\title{
Attitudes and Behaviors of Patients With Rheumatic Diseases During the Early Stages of the COVID-19 Outbreak
}

\author{
Margaret H.Y. Ma ${ }^{1} \mathbb{D}$, Sen Hee Tay ${ }^{1}$ (D) Peter P.M. Cheung ${ }^{1}\left(\mathbb{D}\right.$, Amelia Santosa ${ }^{1}$ (D), \\ Yiong Huak Chan ${ }^{2}$ (D), James W.L. Yip ${ }^{3}$ (D), Anselm Mak ${ }^{1}$ (I) and Manjari Lahiri ${ }^{1}$ (I)
}

\begin{abstract}
Objective. To evaluate attitudes and behaviors of patients with rheumatic diseases during the coronavirus disease 2019 (COVID-19) pandemic.

Methods. An online survey delivered by text message to 4695 patients on follow-up at a tertiary rheumatology center. Latent class analysis was performed on the survey variables.

Results. There were 2239 (47.7\%) who responded to the survey and 3 clusters were identified. Cluster 3 (C3) was defined by patients who were most worried about COVID-19, more likely to wear face masks, and more likely to alter or stop their medications. Patients in C3 were more likely to be female, Malay, and unemployed. Conclusion. We identified 3 clusters with different healthcare beliefs and distinct sociodemographics.
\end{abstract}

Key Indexing Terms: COVID-19, immunosuppressants, rheumatic diseases, survey

The novel coronavirus disease 2019 (COVID-19) has become a global pandemic ${ }^{1}$. Whereas increasing age and comorbidities are proven risk factors for poor clinical outcomes ${ }^{2}$, it is unclear ${ }^{3,4,5,6,7}$ whether patients with rheumatic diseases should be considered high risk due to both disease and immunosuppressive treatments, especially during the early stages of the pandemic. On the other hand, hydroxychloroquine (HCQ) has been used in the treatment of COVID-19 and anti-interleukin 6 has been used for certain patients with cytokine storm syndrome $e^{8,9}$.

COVID-19 has caused increased uncertainty and anxiety among patients, leading to changes in healthcare behaviors ${ }^{10}$. It is important to understand this in the context of rheumatic diseases. As such, we performed a study to explore their attitudes and behaviors, with an aim to identify subgroups of patients with higher needs who may benefit from closer follow-up during this period.

\section{MATERIALS AND METHODS}

Patients seen in our tertiary rheumatology clinic in Singapore between March 1, 2019, and February 29, 2020, with Singapore mobile phones were extracted from our data warehouse using a data anonymization solution

${ }^{I}$ M.H. Ma, MBBS, MRCP, PhD, FAMS, S.H. Tay, MBBS, PhD, P.P. Cheung, MBBS, PhD, FRACP, A. Santosa, MBBS, MRCP, MMed, FAMS, A. Mak, MMed Sc, MBBS, PhD, MD, M. Labiri, MBBS, MD, Senior Consultant, Rheumatology and Assistant Professor, Medicine, Division of Rheumatology, University Medicine Cluster, National University Health System, and Department of Medicine, Yong Loo Lin School of Medicine, National University of Singapore; ${ }^{2}$ Y.H. Chan, PhD, Biostatistics Unit, Yong Loo Lin School of Medicine, National University of Singapore; ${ }^{3}$ J.W. Yip, MBBS, MRCP, FAMS, National University of Singapore Saw Swee Hock School of Public Health, Singapore.

Address correspondence to Dr. M. Labiri, Division of Rheumatology,

University Medicine Cluster, National University Hospital,

IE Kent Ridge Road, NUHS Tower Block, Level 10, Singapore 119228.

Email:manjari_labiri@nubs.edu.sg.

Accepted for publication September 5, 2020. with a unique hashtag key. These patients were sent a 25 -question survey (Supplementary Data 1, available with the online version of this article) through SMS on March 28, 2020, with a secure web-based survey tool (Research Database Capture; REDCap) ${ }^{11}$. A further 2 SMS reminders were sent on March 31, 2020, and April 9, 2020, if the survey was not completed. Data points were aggregated with demographics, diagnosis codes, and prescriptions associated with the rheumatology visit; mobile numbers were removed to preserve anonymity. We used an unbiased, multidimensional approach to identify patients with different healthcare attitudes and behaviors and to see if these clusters predict disease characteristics and medication usage. Latent class analysis (LCA) was performed to determine the appropriate number of clusters and the best fitting models were selected using the Akaike information criterion (AIC) and the Bayesian information criterion $(\mathrm{BIC})^{12}$. A 3-cluster solution was obtained after examining the change in the AIC and the BIC values between adjacent clusters. Twenty-two questionnaire responses were selected as input variables for the LCA. Missing data were input as "unknown" to make up a complete dataset. For survey responses, there were no missing data because the questions were all mandated. This analysis places subjects into clusters, suggested by the questionnaire data, not defined a priori. Differences between clusters were explored using the chi-square test and the Kruskal-Wallis test, with the Mann-Whitney $U$ test for pairwise comparisons and the Bonferroni correction for multiple comparisons. Stata v10 (StataCorp) and SPSS v25 (IBM Corp.) were used. This study was approved by the National Health Group Domain Specific Review Board (NHG DSRB Reference: 2020/00248). Informed consent was not required since it was anonymized.

\section{RESULTS}

Of the 5085 eligible patients, the survey was sent to 4695 individuals with Singapore mobile numbers: 2239 (47.7\%) responded by April 14, 2020 [mean age 53.5 (SD 15.9) yrs, 1567 (70\%) females, 1524 (68.1\%) Chinese]. Four hundred seventy-seven (26.7\%) patients had connective tissue disease (CTD) and 721 (40.3\%) patients had inflammatory arthritis (IA). Two hundred fourteen (9.6\%) patients were taking HCQ alone, 1032 (46.1\%) patients were taking immunosuppressants other than biologics, and 95 (4.2\%) patients were taking biologic or targeted synthetic 
disease-modifying antirheumatic drugs. Survey responders tended to be younger, more likely to be female, non-Chinese, more likely to have IA or CTD, and less likely to have comorbid conditions (Table 1). Forty-four (2.03\%) patients had been tested for SARS-CoV-2 and only 1 patient was positive. Forty-eight $(2.27 \%)$ of the patients' coinhabitants tested positive for SARS-CoV-2.

The majority of patients obtained information on COVID-19 through multiple sources, including television (65.8\%), social media (62.3\%), the Singapore Ministry of Health website and WhatsApp messaging app (61.7\%), newspapers (49.5\%), or friends and family (50.5\%). One hundred sixty-two $(7.4 \%)$ patients had contacted healthcare professionals (HCP) for COVID-19 advice and most (90.74\%) were reassured by their advice. Patients were worried about COVID-19 [median 73 (IQR 50-89) on a 100-point visual analog scale (VAS), with 100 being the most worried] and worried about going out (median 70, IQR 50-81). Seven hundred thirty-three (33.5\%) patients had traveled abroad from December 1, 2019, to the time of the survey and 1020 (47.3\%) had canceled travel plans. There were 1012 (46.4\%) patients who wore face masks while being in good health, despite this being discouraged by the government at the time of the survey. There were 2088 (96\%) patients who sanitized hands more often. During the COVID-19 period,

Table 1. Baseline characteristics.

\begin{tabular}{|c|c|c|c|}
\hline & $\begin{array}{l}\text { Overall Cohort, } \\
\mathrm{n}=5085\end{array}$ & $\begin{array}{c}\text { Survey Responders, } \\
\mathrm{n}=2239\end{array}$ & $\begin{array}{l}\text { Survey Nonresponders, } \\
\mathrm{n}=2846\end{array}$ \\
\hline Age, yrs, mean (SD) & & $53.5(15.9)$ & $59.2(16.0)$ \\
\hline Sex, female & $3450(67.9)$ & $1567(70.0)$ & $1883(66.2)$ \\
\hline \multicolumn{4}{|l|}{ Race } \\
\hline Chinese & $3596(70.7)$ & $1524(68.1)$ & $2072(72.8)$ \\
\hline Malay & $483(9.5)$ & $249(11.1)$ & $234(8.2)$ \\
\hline Indian & $493(9.7)$ & $233(10.4)$ & $260(9.1)$ \\
\hline Other & $512(10.1)$ & $233(10.4)$ & $279(9.8)$ \\
\hline \multicolumn{4}{|l|}{ Diagnosis $^{\dagger}$} \\
\hline CTD & $946(23.5)$ & $477(26.7)$ & $469(21.0)$ \\
\hline IA & $1599(39.8)$ & $721(40.3)$ & $878(39.3)$ \\
\hline Crystal & $483(12.0)$ & $165(9.2)$ & $318(14.2)$ \\
\hline Noninflammatory & $993(24.7)$ & $426(23.8)$ & $567(25.4)$ \\
\hline \multicolumn{4}{|l|}{ Comorbidities* } \\
\hline CVD & $397(8.2)$ & $138(6.4)$ & $259(9.6)$ \\
\hline Hypertension & $306(6.3)$ & $132(6.1)$ & $174(6.4)$ \\
\hline Diabetes mellitus & $337(6.9)$ & $134(6.2)$ & $203(7.5)$ \\
\hline CKD & $161(3.3)$ & $60(2.8)$ & $101(3.7)$ \\
\hline Cancer & $218(4.5)$ & $80(3.7)$ & $138(5.1)$ \\
\hline \multicolumn{4}{|l|}{ Rheumatological medications } \\
\hline None & $1647(32.4)$ & $699(31.2)$ & $948(33.1)$ \\
\hline HCQ alone & $464(9.1)$ & $214(9.6)$ & $250(8.8)$ \\
\hline IS including csDMARD & $2212(43.5)$ & $1032(46.1)$ & $1180(41.4)$ \\
\hline bDMARD or tsDMARD & $189(3.7)$ & $95(4.2)$ & $94(3.3)$ \\
\hline Gout medications & $573(11.3)$ & $199(8.9)$ & $374(13.1)$ \\
\hline \multicolumn{4}{|l|}{ Antihypertensives } \\
\hline None & $3496(68.7)$ & $1604(71.6)$ & $1892(66.5)$ \\
\hline ACEI/ARB & $933(18.4)$ & $387(17.3)$ & $546(19.2)$ \\
\hline Other antihypertensives & $656(12.9)$ & $248(11.1)$ & $408(14.3)$ \\
\hline NSAID or COXIB & $1876(36.9)$ & $855(38.2)$ & $1021(35.9)$ \\
\hline
\end{tabular}

Values are $\mathrm{n}(\%)$ unless otherwise specified. ${ }^{\dagger} \mathrm{CTD}$ : connective tissue disease, includes systemic lupus erythematosus, immune-mediated inflammatory myositis, systemic sclerosis, systemic vasculitis, Sjögren syndrome, mixed CTD, undifferentiated CTD; IA: inflammatory arthritis, includes rheumatoid arthritis, psoriatic arthritis, axial and peripheral spondyloarthropathy, juvenile idiopathic arthritis, adult-onset Still disease, and undifferentiated IA; noninflammatory: includes primary antiphospholipid syndrome, osteoarthritis, osteoporosis, fibromyalgia, soft tissue rheumatism, and miscellaneous conditions. ${ }^{*}$ Comorbidities are based on diagnosis codes and are significantly undercoded as seen by proportion taking antihypertensive drugs; diagnosis codes for hypertension, diabetes, CVD, and CKD combined. ACEI: angiotensin-converting enzyme inhibitor; ARB: angiotensin receptor blocker; bDMARD: biologic disease-modifying antirheumatic drug; CKD: chronic kidney disease; COXIB: cycloxygenase-2 inhibitor; csDMARD: conventional synthetic disease-modifying antirheumatic drug; CVD: cardiovascular disease; HCQ: hydroxychloroquine; IS: immunosuppressant; NSAID: nonsteroidal antiinflammatory drug; tsDMARD: targeted synthetic disease-modifying antirheumatic drug. 
$255(11.7 \%)$ patients had seen their doctors more often than usual. Three hundred sixteen (14.7\%) patients admitted to not taking medications regularly even prior to the pandemic. Since the pandemic, 1991 (94.8\%) patients took their medications with the same regularity or more than before. Of the 109 (5.2\%) patients who took their medications less regularly, $43.3 \%$ did so due to worries about COVID-19. Seven hundred ninety-two (36.8\%) patients and 427 (19.9\%) patients believed that their rheumatic condition and medications would increase their risk of COVID-19, respectively. Three hundred thirteen (14.7\%; $22 \%$ of those receiving HCQ) patients believed their medications would protect them from COVID-19. Only 868 (41\%) patients were up to date with their influenza and pneumococcal vaccinations (Table 2 ).

Three clusters were found using the LCA, classifying C1 (295, $13.2 \%)$, C2 (703, 31.4\%), and C3 (1241, 55.4\%). Discriminant analysis showed that $97.7 \%$ of the subjects were correctly classified with a cross-validation of $97.3 \%$ (Supplementary Figure 1 , available with the online version of this article). Group C3 was the most worried about COVID-19 [median VAS 86 (IQR 78-97)] vs Group C2: 52 (IQR 50-63), $P<0.001$, and vs Group C1: 20 (IQR 10-29), $P<0.001]$. This group had more contact with HCP compared to $\mathrm{C} 1(9 \%$ vs $6 \%, P=0.01$ ), with more patients wearing face masks ( $53 \%$ vs $28 \%, P<0.001)$. They also decreased their social activities ( $80 \%$ vs $68 \%, P<0.001)$. They were most worried that their condition ( $43 \%$ vs $20 \%, P<0.001$ ) or medications $(22 \%$ vs $13 \%, P<0.001)$ would put them at higher risk of being infected. C3 also altered or stopped their medications more during COVID-19 ( $11 \%$ vs $7 \%, P=0.002)$ and took medication less regularly before COVID-19 (20\% vs $16 \%, P=0.02)$. C3 obtained less information from newspapers ( $47 \%$ vs $54 \%, P=0.03$ ), and fewer were up to date with vaccinations ( $37 \%$ vs $44 \%, P=0.006$; Table 2 ).

On examination of the demographic and clinical profiles of the generated clusters, patients in $\mathrm{C} 3$ were more likely to be female, of Malay race, and unemployed. Rheumatic conditions and medication type were not different among the 3 clusters (Table 3). In a subset analysis of patients with IA or CTD and/ or on immunomodulatory medications, patients with CTD were more likely to belong to $\mathrm{C} 3$, although other descriptors of group membership remained the same.

\section{DISCUSSION}

To our knowledge, our study is the first to survey a wide spectrum of multiethnic patients with rheumatic diseases about COVID-19. We identified a large subset of patients who were

Table 2. Latent class analysis generation of 3 clusters.

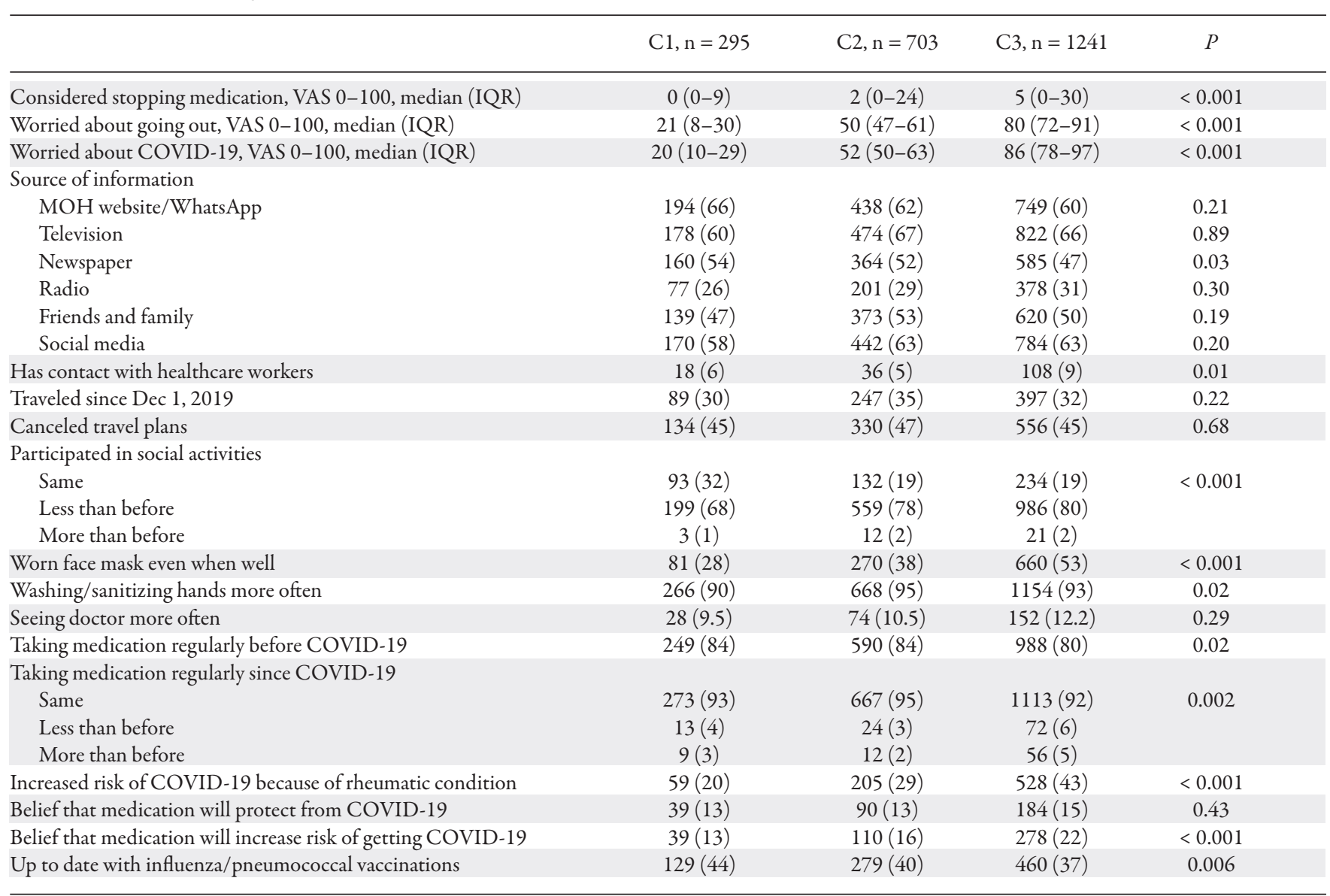

Values are $\mathrm{n}(\%)$ unless otherwise specified. Cluster groups: C1/C2/C3 used the whole cohort of patients who had responded to questionnaire. Differences between clusters were tested using Kruskal-Wallis test for continuous measures and Pearson chi-square test for categorical measures. MOH: Ministry of Health. 


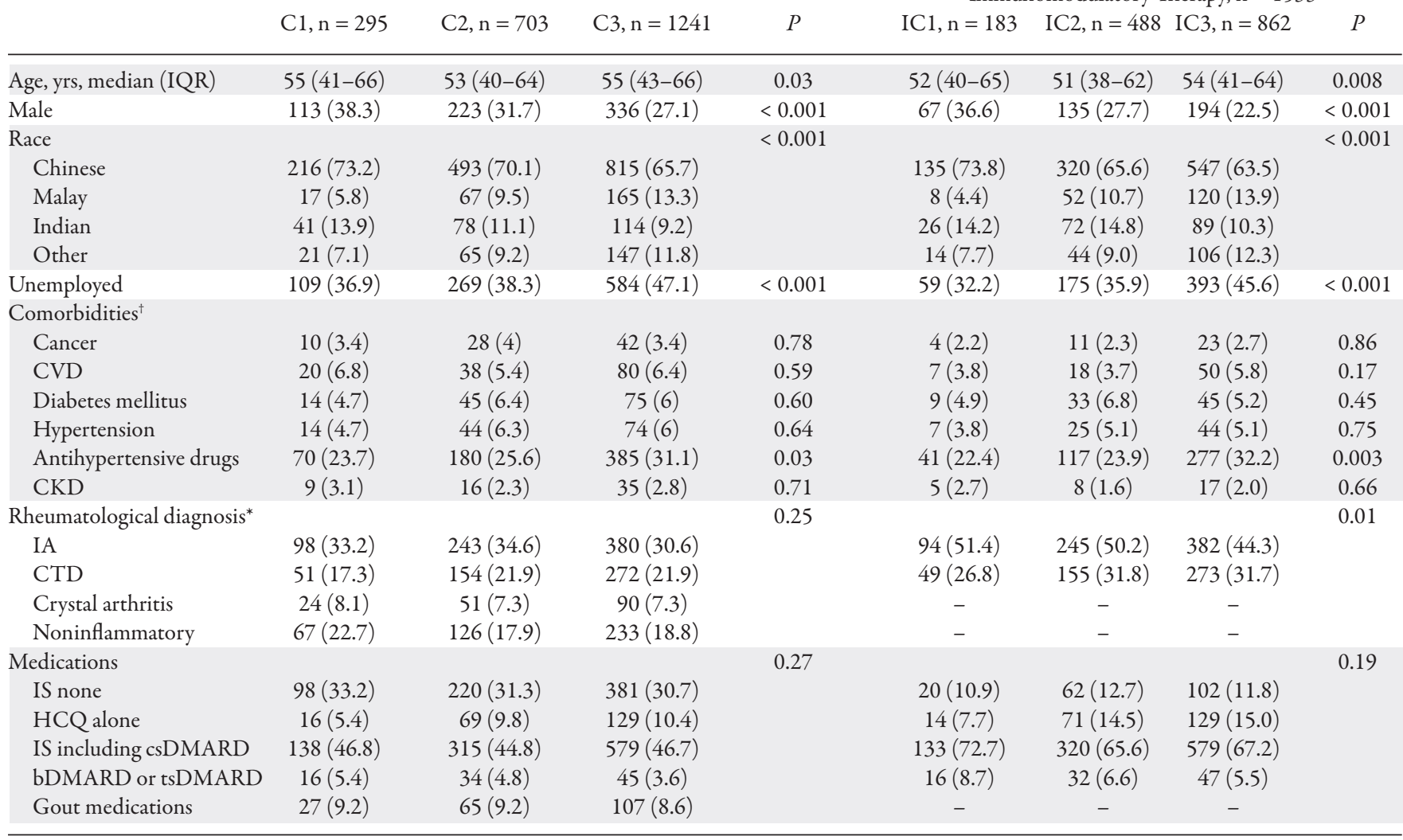

Values are $\mathrm{n}(\%)$ unless otherwise specified. Cluster groups: C1/C2/C3 used the whole cohort of patients who had responded to questionnaire. A subgroup analysis was carried out on patients with a diagnosis of connective tissue disease, inflammatory arthritis, and/or on immunosuppressive agents (IC1/IC2/IC3). Differences between clusters were tested using Kruskal-Wallis test for continuous measures and Pearson chi-square test for categorical measures. ${ }^{*}$ Unknown $=450 .{ }^{\dagger} \mathrm{CTD}$ : Connective tissue disease, includes systemic lupus erythematosus, immune mediated inflammatory myositis, systemic sclerosis, systemic vasculitis, Sjögren syndrome, mixed CTD, undifferentiated CTD; IA: inflammatory arthritis, includes rheumatoid arthritis, psoriatic arthritis, axial and peripheral spondyloarthropathy, juvenile idiopathic arthritis, adult-onset Still disease, and undifferentiated IA; noninflammatory: includes primary antiphospholipid syndrome, osteoarthritis, osteoporosis, fibromyalgia, soft tissue rheumatism, and miscellaneous conditions. bDMARD: biologic disease-modifying antirheumatic drug; C1, C2, C3: cluster 1, 2, 3; CKD: chronic kidney disease; csDMARD: conventional synthetic disease-modifying antirheumatic drug; CVD: cardiovascular disease; HCQ: hydroxychloroquine; IS: immunosuppressant; tsDMARD: targeted synthetic disease-modifying antirheumatic drug.

more worried about COVID-19 because of their underlying disease and medications. These patients were more likely to contact HCP, modify their social behaviors, and alter/stop medications. This group had a lower proportion of patients reading the newspaper, being adherent to their medications, or being up to date with their vaccinations. This cluster represented a vulnerable sociodemographic group, with significantly higher unemployment, more women, and belonging to an ethnic minority, but who were not different in the distribution of rheumatic diseases or immunosuppressive medications. Patients with such disadvantaged sociodemographic profiles are also similarly seen in other cohorts of rheumatic diseases [e.g., Lupus in Minority: NAture vs Nurture (LUMINA) cohort $]^{13}$. Patients clustered in C3 may benefit from more rheumatology services.

In a US survey (response rate 7.5\%), 530 patients with rheumatic diseases were significantly worried about risk of COVID-19, with $14 \%$ self-changing their medication or dose ${ }^{14}$. In our study, medication adjustments were observed only in 5\% of the patients, possibly due to the patients' perception that their rheumatic disease itself posed a greater risk. However, increased handwashing, less travel, and fewer social activities among the general US population were similar to our findings ${ }^{10}$.

Our study has several strengths. We were able to distribute the survey link through SMS to $92 \%$ of the patients with a high response rate. We used a unique cluster analysis to avoid bias from testing for multiple outcomes. Our patients include a multiethnic population, representative of Singapore, which is predominantly Chinese (74\%), followed by Malays (13\%) and Indians $(9 \%)^{15}$, allowing us to study racial and cultural differences in attitudes and behavior. However, while associations exist between sociodemographics and perceived risk of COVID-19, causation cannot be demonstrated due to the study design. We were limited by the cross-sectional nature of our survey, which did not allow us to determine the longitudinal stability of our clusters. Although not included in our survey, patient-perceived disease activity may have influenced their attitudes during and 
toward COVID-19 and would be thus informative to include in future studies. This survey was conducted mostly before stricter lockdown measures were implemented in Singapore on April 7, 2020; behavior may since have changed.

The pathological and psychological effect of COVID-19 on patients with rheumatic diseases likely exists as a consequence of their immunosuppressive state and inherently heightened anxiety and depression levels ${ }^{16}$. While almost $20 \%$ of respondents were concerned that their medications would increase their risk for COVID-19, only a small proportion sought advice from their physicians. An effective platform for patients to express their concerns in the context of COVID-19 will be fundamental to prevent unnecessary or even harmful unilateral changes of treatment by patients. Some rheumatological societies have published recommendations to address these issues; however, these societies additionally recommend cancellation of routine visits $^{17,18,19}$. Rheumatologists will need to find novel ways to engage their patients proactively to allay anxiety. We have identified a subgroup of patients where these efforts should be most targeted.

\section{ACKNOWLEDGMENT}

We would like to thank the team at the NUHS Academic informatics office (AIO) for their assistance in data acquisition.

\section{ONLINE SUPPLEMENT}

Supplementary material accompanies the online version of this article.

\section{REFERENCES}

1. Zhu N, Zhang D, Wang W, Li XW, Yang B, Song JD, et al; China Novel Coronavirus Investigating and Research Team. A novel coronavirus from patients with pneumonia in China, 2019. N Engl J Med 2020;382:727-33.

2. Wang D, Hu B, Hu C, Zhu F, Liu X, Zhang J, et al. Clinical characteristics of 138 hospitalized patients with 2019 novel coronavirus-infected pneumonia in Wuhan, China. JAMA 2020;323:1061-9.

3. Gianfrancesco MA, Hyrich KL, Gossec L, Strangeld A, Carmona L, Mateus EF, et al; COVID-19 Global Rheumatology Alliance Steering Committee. Rheumatic disease and COVID-19: initial data from the COVID-19 Global Rheumatology Alliance provider registries. Lancet Rheumatol 2020;2:250-3.

4. Monti S, Balduzzi S, Delvino P, Bellis E, Quadrelli V, Montecucco C, et al. Clinical course of COVID-19 in a series of patients with chronic arthritis treated with immunosuppressive targeted therapies. Ann Rheum Dis 2020;79:667-8.

5. Mathian A, Mahevas M, Rohmer J, Roumier M, Cohen-Aubart F, Amador-Borrero B, et al. Clinical course of coronavirus disease 2019 (COVID-19) in a series of 17 patients with systemic lupus erythematosus under long-term treatment with hydroxychloroquine. Ann Rheum Dis 2020;79:837-9.
6. Favalli EG, Ingegnoli F, Cimaz R, Caporali R. What is the true incidence of COVID-19 in patients with rheumatic diseases? Ann Rheum Dis 2020 April 16 (Epub ahead of print).

7. Haberman R, Axelrad J, Chen A, Castillo R, Yan D, Izmilry P, et al. Covid-19 in immune-mediated inflammatory diseases - case series from New York. N Engl J Med 2020;383:85-8.

8. Kim AH, Sparks JA, Liew JW, Putman M, Berenbaum F, Duarte-Garcia A, et al. A rush to judgment? Rapid reporting and dissemination of results and its consequences regarding the use of hydroxychloroquine for COVID-19. Ann Intern Med 2020;172:819-21.

9. Mehta P, McAuley DF, Brown M, Sanchez E, Tattersall R, Manson J, et al; HLH Across Speciality Collaboration, UK. COVID-19: consider cytokine storm syndromes and immunosuppression. Lancet 2020;395:1033-4.

10. Nelson LM, Simard JF, Oluyomi A, Nava V, Rosas L, Bondy M, et al. US Public concerns about the COVID-19 pandemic from results of a survey given via social media. JAMA Intern Med 2020; 180:1020-2.

11. Harris PA, Taylor R, Thielke R, Payne J, Gonzalez N, Conde J. Research electronic data capture (REDCap)-a metadata-driven methodology and workflow process for providing translational research informatics support. J Biomed Inform 2009; 42:377-81.

12. Collins LM, Lanza ST. Latent class and latent transition analysis for the social, behavioural, and health sciences. New York: Wiley; 2010.

13. Uribe AG, McGwin G Jr, Reveille JD, Alarcón GS. What have we learned from a 10-year experience with the LUMINA (Lupus in Minorities; Nature vs. nurture) cohort? Where are we heading? Autoimmun Rev 2004;3:321-9.

14. Michaud K, Wipfler K, Shaw Y, Simon T, Cornish A, England B, et al. Experiences of patients with rheumatic diseases in the US during early days of the COVID-19 pandemic. ACR Open Rheumatol 2020;2:335-43.

15. Department of Statistics, Singapore. Population dashboard. [Internet. Accessed September 7, 2020.] Available from: www. singstat.gov.sg/find-data/search-by-theme/population/ population-and-population-structure/visualising-data/ population-dashboard

16. Ho RC, Fu EH, Chua AN, Cheak A, Mak A. Clinical and psychosocial factors associated with depression and anxiety in Singaporean patients with rheumatoid arthritis. Int J Rheum Dis 2011;14:37-47.

17. American College of Rheumatology COVID-19 Clinical Guidance Task Force. COVID-19 clinical guidance for adult patients with rheumatic diseases. [Internet. Accessed October 5, 2020.] Available from: www.rheumatology.org/Portals/0/Files/ACR-COVID-19Clinical-Guidance-Summary-Patients-with-Rheumatic-Diseases.pdf

18. EULAR. EULAR Guidance for patients COVID-19 outbreak. [Internet. Accessed September 7, 2020.] Available from: www.eular. org/eular_guidance_for_patients_covid19_outbreak.cfm

19. British Society for Rheumatology. COVID-19 guidance. [Internet. Accessed September 7, 2020.] Available from: www.rheumatology. org.uk/news-policy/details/Covid19-Coronavirus-update-members 